Pacific Journal of Mathematics

THE FINE SPECTRA FOR WEIGHTED MEAN OPERATORS 


\section{THE FINE SPECTRA FOR WEIGHTED MEAN OPERATORS}

\section{B. E. RHOADES}

In a recent paper [5] the fine spectra of integer powers of the Cesàro matrix were computed. In this paper the fine spectra of weighted mean methods are determined. In most cases investigated, the interior points belong to $\mathrm{III}_{1}$, the boundary points, except 1 , belong to $\mathrm{II}_{2}$, and 1 and any isolated points belong to $\mathrm{III}_{3}$, where $\mathrm{III}_{1}, \mathrm{II}_{2}$, and $\mathrm{III}_{3}$ are portions of the state space as described in [3].

From Goldberg [3], if $T \in B(X), X$ a Banach space, then there are three possibilities for $R(T)$, the range of $T$ :

(I) $R(T)=X$,

(II) $\overline{R(T)}=X$, but $R(T) \neq X$, and

(III) $\overline{R(T)} \neq X$, and three possibilities for $T^{-1}$

(1) $T^{-1}$ exists and is continuous,

(2) $T^{-1}$ exists but is discontinuous,

(3) $T^{-1}$ does not exist.

A weighted mean matrix $A$ is a lower triangular matrix with entries $a_{n k}=p_{k} / P_{n}$, where $p_{0}>0, p_{n} \geq 0$ for $n>0$, and $P_{n}=\sum_{k=0}^{n} p_{k}$. The necessary and sufficient condition for the regularity of $A$ is that $\lim P_{n}=$ $\infty$.

In [2] it was shown that, for any regular weighted mean matrix $A$, the spectrum, $\sigma(A)$, contains the set $\left\{\lambda|| \lambda-(2-\delta)^{-1} \mid \leq(1-\delta) /(2-\delta)\right\}$ $\cup S$, and is contained in the set $\left\{\lambda|| \lambda-(2-\gamma)^{-1} \mid \leq(1-\gamma) /(2-\gamma)\right\}$ $\cup \underline{S, \text { where } \delta}=\lim \sup p_{n} / P_{n}, \quad \gamma=\lim \inf p_{n} / P_{n}$, and $S$ $=\overline{\left\{p_{n} / P_{n} \mid n \geq 0\right\}}$

We shall first consider those regular weighted mean methods for which $\delta=\gamma$, i.e., for which the main diagonal entries converge.

THEOREM 1. Let $A$ be a regular weighted mean method such that $\delta=\lim p_{n} / p_{n}$ exists. If $\lambda$ satisfies $\left|\lambda-(2-\delta)^{-1}\right|<(1-\delta) /(2-\delta)$ and $\lambda \notin S$, then $\lambda \in \mathrm{III}_{1} \sigma(A)$; i.e., $\lambda$ is a point of $\sigma(A)$ for which $\overline{R(T)} \neq X$ and $T^{-1}$ exists and is continuous.

Proof. First of all $\lambda I-A$ is a triangle, hence 1-1. Therefore $\lambda I-A$ $\in 1 \cup 2$. 
Consider the adjoint matrix $T^{*}=\lambda I-A^{*}$. Since $A$ is regular, $A^{*} \in$ $B(l)$ with entries $a_{00}^{*}=\chi(A)=\lim _{n} A e-\Sigma_{k} \lim _{n} a_{n k}=1, a_{n 0}^{*}=a_{0 n}^{*}=0$ for $n>0$ and $a_{n k}^{*}=a_{k-1, n-1}$ for $n, k>0$, where $e=\{1,1,1, \ldots\}$.

Suppose $T^{*} x=0$. Then

$$
(\lambda-1) x_{0}=0
$$

and

$$
\left(\lambda-\frac{p_{n-1}}{P_{n-1}}\right) x_{n}-\sum_{k=n+1}^{\infty} a_{n k}^{*} x_{k}=0 \text { for } n>0 .
$$

Thus $x_{0}=0$ and, from (1), $x_{1}$ is arbitrary and, with $c_{n}=p_{n} / P_{n}$,

$$
\begin{aligned}
x_{n} & =\frac{p_{n-1} x_{1}}{p_{0} \lambda^{n-1}} \prod_{j=0}^{n-2}\left(\lambda-c_{j}\right)=\frac{p_{n-1}}{p_{0}} x_{1} \prod_{j=0}^{n-2}\left(1-\frac{c_{j}}{\lambda}\right) \\
& =\left(1-\frac{1}{\lambda}\right) \frac{p_{n-1} x_{1}}{P_{n-2}} \prod_{j=1}^{n-2}\left(1+\left(1-\frac{1}{\lambda}\right) \frac{p_{j}}{P_{j-1}}\right) .
\end{aligned}
$$

if

Now $\left|1+\left(1-\frac{1}{\lambda}\right) p_{j} / P_{j-1}\right|<1$ for all $j$ sufficiently large if and only

$$
\left(1+(1+\alpha) \frac{p_{j}}{P_{j-1}}\right)^{2}+\left(\beta \frac{p_{j}}{P_{j-1}}\right)^{2}<1, \text { where }-\frac{1}{\lambda}=\alpha+i \beta .
$$

Case I. Assume at most a finite number of the $p_{k}$ are zero. Then the above inequality is equivalent to

$$
2(1+\alpha)+\left((1+\alpha)^{2}+\beta^{2}\right)\left(p_{j} / P_{j-1}\right)<0
$$

for all $j$ sufficiently large. The above inequality will be true for all $j$ sufficiently large if $2(1+\alpha)+\left((1+\alpha)^{2}+\beta^{2}\right) \delta /(1-\delta)<0$, which is equivalent to $\left|\lambda-(2-\delta)^{-1}\right|<(1-\delta) /(2-\delta)$.

Let $z_{n}=\prod_{j=1}^{n-2}\left(1+(1-1 / \lambda) p_{j} / P_{j-1}\right)$. Then

$$
\left|z_{n+1} / z_{n}\right|=\left|1+(1-1 / \lambda) p_{n-1} / P_{n-2}\right| \text {. }
$$

From the hypothesis on $\lambda$, and the discussion in the preceding paragraph,

$$
\left|1+\left(1-\frac{1}{\lambda}\right) \frac{p_{n-1}}{P_{n-2}}\right| \leq \xi<1 .
$$

for all $n$ sufficiently large, and $\Sigma\left|z_{n}\right|$ is convergent.

Since $\left|(1-1 / \lambda) p_{n-1} x_{1} / P_{n-2}\right|$ is bounded, it follows that $\Sigma\left|x_{n}\right|$ is convergent, so that $\left(\lambda I-A^{*}\right) x=0$ has nonzero solutions.

By [3, Theorem II 3.7], $\lambda I-A$ does not have dense range. Therefore $\lambda I-A \in \mathrm{III}$ and hence $\lambda I-A \in \mathrm{III}_{1} \cup \mathrm{III}_{2}$. 
To verify that $\lambda I-A \in \mathrm{III}_{1}$ it is sufficient, from [3, Theorem II 3.11], to show that $\lambda I-A^{*}$ is onto.

Suppose $y=\left(\lambda I-A^{*}\right) x, x, y \in l$. Then $(\lambda-1) x_{0}=y_{0}$ and

$$
\left(\lambda-c_{n-1}\right) x_{n}-\sum_{k=n+1}^{\infty} p_{n-1} x_{k} / P_{k-1}=y_{n}, \quad n>0 .
$$

Choose $x_{1}=0$ and solve for $x$ in terms of $y$ to get

$$
\begin{gathered}
-p_{0} \sum_{k=2}^{\infty} x_{k} / P_{k-1}=y_{1} \\
\left(\lambda-c_{n-1}\right) x_{n}=y_{n}+p_{n-1} \sum_{k=n+1}^{\infty} x_{k} / P_{k-1} .
\end{gathered}
$$

For example, substituting (5) into (6), with $n=2$, yields

$$
\left(\lambda-c_{1}\right) x_{2}=y_{2}+p_{1} \sum_{k=3}^{\infty} x_{k} / P_{k-1}=y_{2}+p_{1}\left(\sum_{k=2}^{\infty} x_{k} / P_{k-1}-x_{2} / P_{1}\right),
$$

so that $x_{2}=\left(y_{2}-p_{1} y_{1} / p_{0}\right) / \lambda$.

Continuing this process, if $B$ is a lower triangular matrix defined by $B y=x$, then $B$ has entries

$$
\begin{aligned}
& b_{00}=\frac{1}{\lambda-1}, \quad b_{n n}=\frac{1}{\lambda}, \quad n>1, \\
& b_{21}=\frac{-p_{1}}{\lambda p_{0}}, \quad b_{n, n-1}=-\frac{p_{n-1}}{P_{n-2} \lambda^{2}}, \quad n>2, \\
& b_{n 1}=-\frac{p_{n-1}}{\lambda p_{0}} \prod_{j=1}^{n-2}\left(1-\frac{c_{j}}{\lambda}\right), \quad n>2, \\
& b_{n k}=-\frac{p_{n-1}}{\lambda^{2} P_{k-1}} \prod_{j=k}^{n-2}\left(1-\frac{c_{j}}{\lambda}\right), \quad 1<k<n-1,
\end{aligned}
$$

and $b_{n k}=0$ otherwise.

To show that $B \in B(l)$ it is sufficient to show that $\Sigma_{n}\left|b_{n k}\right|$ is finite, independent of $k$.

$\Sigma_{n}\left|b_{n 0}=1 /\right| \lambda-1 \mid$. We may write $1-c_{j} / \lambda=1-p_{j} / \lambda P_{j}=$ $\left(P_{j-1} / P_{j}\right)\left(1+(1-1 / \lambda) p_{j} / P_{j-1}\right)$. Also, $\sup _{n>1}\left|p_{n-1} / P_{n-2}\right| \leq M<\infty$. Therefore

$$
\sum_{n}\left|b_{n 1}\right| \leq \frac{1}{|\lambda|}\left(M+M \sum_{n=3}^{\infty} \prod_{j=1}^{n-2}\left|1+\left(1-\frac{1}{\lambda}\right) \frac{p_{j}}{P_{j-1}}\right|\right),
$$


and, for $k>1$,

$$
\sum_{n}\left|b_{n k}\right| \leq \frac{1}{|\lambda|}+\frac{M}{|\lambda| 2}+\frac{M}{|\lambda| 2} \sum_{n=k+2}^{\infty} \prod_{j=k}^{n-2}\left|1+\left(1-\frac{1}{\lambda}\right) \frac{p_{j}}{P_{j-1}}\right| .
$$

Since $k>1$, the series in the second inequality is dominated by the series in the first inequality which, from (3), is absolutely convergent. Therefore $\|B\|_{1}<\infty$.

Since $(\lambda I-A)^{-1}$ is bounded, it is continuous, and $\lambda \in \operatorname{III}_{1} \sigma(A)$.

Case II. Suppose an infinite number of the $p_{k}$ are zero. Since $\lim _{n} p_{n}$ $=\infty$, there are an infinite number of nonzero $p_{k}$. Denote these by $\left\{p_{n_{k}}\right\}$.

From (2) for $n \neq 1+n_{k}, x_{n}=0$. Otherwise,

$$
x_{1+n_{k}}=(1-1 / \lambda) \frac{p_{n_{k}}}{P_{n_{k}-1}} \prod_{j=1}^{r}\left(1-(1-1 / \lambda) \frac{p_{n_{j}}}{P_{n_{j}-1}}\right) .
$$

Now apply the same analysis as in Case I to verify that $\sum\left|z_{n}\right|$ converges, and hence $\lambda I-A^{*}$ has nonzero solutions.

To show that $\lambda I-A^{*}$ is onto, the presence of an infinite number of $p_{k}=0$ merely introduces more zero entries in $B$. For the non-zero entries, the same argument as Case I applies.

THEOREM 2. Let $A$ be a regular weighted mean method such that $\delta=\lim p_{n} / P_{n}$ exists, and $\delta<1$. Suppose no diagonal entry of $A$ occurs an infinite number of times. If $\lambda=\delta$, or $\lambda=a_{n n}, n=1,2,3, \ldots$ and $\delta /(2-\delta)<\lambda<1$, then $\lambda \in \operatorname{III}_{1} \sigma(A)$.

Proof. First assume that $A$ has distinct diagonal entries, and fix $j \geq 1$. Then the system $\left(a_{j j} I-A\right) x=0$ implies $x_{k}=0$ for $k=0,1, \ldots, j-1$, and, for $n \geq j$,

$$
\left(a_{j j}-a_{n n}\right) x_{n}-\sum_{k=0}^{n-1} a_{n k} x_{k}=0 .
$$

The above system yields the recursion relation

$$
x_{n+1}=p_{J} P_{n} x_{n} / P_{j} P_{n+1}\left(c_{j}-c_{n+1}\right),
$$

which can be solved for $x_{n}$ to yield

$$
x_{j+m}=\frac{P_{j} x_{j} c_{j}^{m}}{P_{j+m} \prod_{i=1}^{m}\left(c_{j}-c_{j+i}\right)}=x_{j} \prod_{i=1}^{m}\left(\frac{1-c_{j+i}}{1-c_{j+i} / c_{j}}\right) .
$$


Since $\left(1-c_{j+i}\right) /\left(1-c_{j+i} / c_{j}\right)=\left(P_{j+i}-p_{j+i}\right) /\left(P_{j+i}-p_{j+i} / c_{j}\right)=$ $P_{j+i-1} /\left(P_{j+i-1}+\left(1-1 / c_{j}\right) p_{j+i}\right)=\left(1+\left(1-1 / c_{j}\right) p_{j+i} / P_{j+l-1}\right)^{-1}$,

$$
x_{j+m}=x_{j} / \prod_{i=1}^{m}\left(1+\left(1-1 / c_{J}\right) p_{j+i} / P_{J+i-1}\right) .
$$

Since $0<c_{j}<1$, the argument of Theorem 1 implies that

$$
\left|1+\left(1-1 / c_{j}\right) p_{j+i} / P_{j+i-1}\right| \leq \xi<1
$$

for all $i$ sufficiently large. Therefore $x \in c$ implies $x=0$ and $a_{J j} I-A$ is 1-1, so that $c_{j} I-A \in 1 \cup 2$.

Clearly $c_{j} I-A \in$ III. It remains to show that $c_{j} I-A^{*}$ is onto.

Suppose $\left(c_{J} I-A^{*}\right) x=y, x, y \in l$. By choosing $x_{j+1}=0$ we can solve for $x_{0}, \cdots, x_{j}$ in terms of $y_{0}, \cdots, y_{j+1}$. As in Theorem 1, the remaining equations can be written in the form $x=B y$, where the nonzero entries of $B$ are

$$
b_{j+m, j+m}=1 / c_{J}, \quad m>1 ;
$$

$$
\begin{gathered}
b_{j+2, j+1}=-p_{j+1} / c_{j} p_{j} ; b_{j+m, j+m-1}=-p_{j+m-1} / c_{j}^{2} P_{j+m-2}, \quad m>2 ; \\
b_{j+m, j+k}=-\frac{p_{j+m-1}}{c_{j}^{2} P_{j+k-1}} \prod_{i=j+k}^{j+m-2}\left(1-\frac{c_{i}}{c_{j}}\right), 1<k<m-1, \quad m>2 ; \\
b_{j+m, j+1}=-\frac{p_{j+m-1}}{c_{j} p_{j}} \prod_{i=j+1}^{j+m-2}\left(1-\frac{c_{l}}{c_{j}}\right), \quad m>2 .
\end{gathered}
$$

From (8),

$$
\sum_{n=j+1}^{\infty}\left|b_{n, j+1}\right|=\frac{p_{J+1}}{c_{j} p_{j}}+\frac{1}{c_{j} p_{j}} \sum_{n=j+3}^{\infty} p_{n-1} \prod_{i=j+1}^{n-2}\left|1-\frac{c_{i}}{c_{j}}\right| .
$$

For $m>1$,

$$
\sum_{n=m+j}^{\infty}\left|b_{n, m+j}\right|=\frac{1}{c_{j}}+\frac{p_{j+m}}{c_{j}^{2} P_{j+m-1}}+\frac{1}{c_{j}^{2}} \sum_{n=m+j+2}^{\infty} \frac{p_{n-1}}{P_{j+m-1}} \prod_{i=j+m}^{n-2}\left|1-\frac{c_{i}}{c_{j}}\right| .
$$

Since $p_{j+m} / P_{j+m-1}$ is bounded, and $p_{j} / P_{j+m-1} \leq 1$ for each $m>1$, to show that $\|B\|_{1}$ is finite, it is sufficient to show that the series in (9) converges. We may write

$$
\frac{p_{n-1}}{p_{j}}=\frac{p_{n-1} P_{n-2} P_{n-3} \cdots P_{j}}{P_{n-2} P_{n-3} \cdots P_{j} p_{j}}=\frac{p_{n-1}}{P_{n-2}} \cdot \frac{1}{\left(1-c_{n-2}\right) \cdots\left(1-c_{j+1}\right) c_{j}} .
$$


Substituting in (9) the series then becomes

$$
\frac{1}{c_{j}^{2}} \sum_{n=j+3}^{\infty} \frac{p_{n-1}}{P_{n-2}} \prod_{i=j+1}^{n-2}\left|\frac{1-c_{i} / c_{j}}{1-c_{i}}\right| .
$$

Note that

$$
\begin{aligned}
\frac{1-c_{i} / c_{j}}{1-c_{i}} & =\frac{P_{\imath}-p_{i} / c_{j}}{P_{i}-p_{i}}=\frac{p_{i}}{P_{i-1}}+1-\frac{p_{i}}{c_{j} P_{i-1}} \\
& =1+\left(1-\frac{1}{c_{j}}\right) \frac{p_{i}}{P_{i-1}} .
\end{aligned}
$$

From the hypothesis on $\lambda,(3)$ is satisfied for all $i$ sufficiently large, and the series in (9) is absolutely convergent.

Suppose $A$ does not have distinct diagonal entries. The restriction on $\lambda$ guarantees that no zero diagonal entries are being considered. Let $c_{j} \neq 0$ be any diagonal entry which occurs more than once, and let $k, r$ denote, respectively, the smallest and largest integers for which $c_{j}=c_{k}=c_{r}$. From (7) it follows that $x_{n}=0$ for $n \geq r$. Also, $x_{n}=0$ for $0 \leq n<k$. Therefore the system $\left(c_{j} I-A\right) x=0$ becomes

$$
\left(c_{j}-c_{n}\right) x_{n}-\sum_{i=j}^{n-1} a_{n i} x_{i}=0, \quad k<n \leq r .
$$

Case I. $r=k+1$. Then (11) reduces to the single equation

$$
\left(c_{j}-c_{k+1}\right) x_{j+1}-a_{k+1, k} x_{k}=0,
$$

which implies $x_{k}=0$, since $c_{j}=c_{r}=c_{k+1}$, and $p_{j} \neq 0$. Therefore $x=0$.

Case II. $r>k+1$. From (11) one obtains the recursion formula $x_{n}=P_{n+1}\left(c_{j}-c_{n+1}\right) x_{n+1} / c_{j} P_{n}, k<n<r$. Since $x_{d} \mathrm{r}=0$ it then follows that $x_{n}=0$ for $k<n<r$. Using (11) with $n=k+1$ yields $x_{k}=0$ and so again $x=0$.

To show that $c_{j} I-A^{*}$ is onto, suppose $\left(c_{j} I-A^{*}\right) x=y, x, y \in l$. By choosing $x_{j+1}=0$ we can solve for $x_{0}, x_{1}, \ldots, x_{j}$ in terms of $y_{0}, y_{1}, \ldots, y_{j+1}$. As in Theorem 1 the remaining equations can be written in the form $x=B y$, where the entries of $B$ are as in (8), with the other entries of $B$ clearly zero.

Since $k \leq j \leq r$, there are two cases to consider.

Case I. $j=r$. Then the proof proceeds exactly as the argument following (8). 
Case II. $j<r$. Then, from (8), $b_{j+m, j+k}=b_{j+m, j+1}=0$ at least for $m \geq r-j+2$. If there are other values of $n, j<n<r$ for which $c_{n}=c_{j}$, then additional entries of $B$ will be zero. These zero entries do not affect the validity of the argument showing that (9) converges.

If $\delta=0$, then 0 does not lie inside the disc, and so it is not considered in this theorem.

Let $\lambda=\delta>0$. If $a_{n n} \neq \delta$ for each $n \geq 1$, all $i$ sufficiently large, then the argument of Theorem 1 applies and $\delta I-A \in$ III $_{1}$. If $a_{n n}=\delta$ for some $n$, then the proof of Theorem 2 applies, with $c_{J}$ replaced by $\delta$, and, again, $\delta I-A \in \mathrm{III}_{1}$.

Therefore, in all cases, $c_{j} I-A \in 1 \cup 2$.

THEOREM 3. Let $A$ be a regular weighted mean method such that $\delta=\lim p_{n} / P_{n}$ exists and $p_{n} / P_{n} \geq \delta$ for all $n$ sufficiently large. If $\lambda$ satisfies $\left|\lambda-(2-\delta)^{-1}\right|=(1-\delta) /(2-\delta), \lambda \neq 1, \delta /(2-\delta)$, then $\lambda \in \mathrm{II}_{2} \sigma(A)$.

Proof. Fix $\lambda \neq 1, \delta /(2-\delta)$, and satisfying $\left|\lambda-(2-\delta)^{-1}\right|=$ $(1-\delta) /(2-\delta)$. Since $\lambda I-A$ is a triangle, it is $1-1$ and $\lambda I-A \in 1 \cup 2$.

Now consider $\left(\lambda I-A^{*}\right) x=0$. As in Theorem $1, x_{0}=0, x_{1}$ is arbitrary and $\left\{x_{n}\right\}$ satisfies (2) for all $n>0$. From the hypothesis there exists a positive integer $N$ such that $n \geq N$ implies $c_{n} \geq \delta$. This fact, together with the condition on $\lambda$, implies that $\left|1+(1-1 / \lambda) p_{n} / P_{n-1}\right| \geq 1$ for $n \geq N$. Thus $\left|x_{n}\right| \geq c p_{n-1} / P_{n-2}$ for $n \geq N$, where $c$ is a constant independent of $n$. We may write

$$
\begin{aligned}
p_{n-1} / P_{n-2} & =\left(p_{n-1} / P_{n-1}\right)\left(p_{n-1} / P_{n-2}\right) \\
& =\left(p_{n-1} / P_{n-1}\right)\left(1+p_{n-1} / P_{n-2}\right) \geq p_{n-1} / P_{n-1} .
\end{aligned}
$$

From [4, p. 290], $\Sigma p_{n} / P_{n}$ diverges, so $\left\{x_{n}\right\} \in l$ implies $x_{1}=0$, hence $x=0$ and $\lambda I-A^{*} \in 1 \cup 2$. Since $\lambda \in \sigma(A)$, and $\lambda \neq 1, \delta /(2-\delta)$, $\lambda \in \mathrm{II}_{2} \sigma(A)$.

THEOREM 4. Let $A$ be a regular weighted mean method. Then $1 \in$ $\mathrm{III}_{3} \sigma(A)$.

Proof. Since $(I-A) e=0, I-A$ is not 1-1 and hence $I-A \in 3$. It remains to show that $\overline{R(I-A)} \neq c$. Let $z \in c$ such that $z_{0} \neq 0$. Then $\|(I-A) x-z\| \geq\left|z_{0}\right|>\left|z_{0}\right| / 2$ for all $x \in c$. Therefore $z \notin \overline{R(I-A)}$.

THEOREM 5. Let $A$ be a regular weighted mean method with $\gamma=$ $\lim \inf p_{n} / P_{n}$. If there exists values of $n$ such that $0 \leq c_{n} \leq \gamma /(2-\gamma)$, then $\lambda=c_{n}$ implies $\lambda \in \mathrm{III}_{3} \sigma(A)$. 
Proof. Let $c_{k}$ be any diagonal entry satisfying $0<c_{k} \leq \gamma /(2-\gamma)$. Let $j$ be the smallest integer such that $c_{j}=c_{k}$. Since $c_{0}=1, j>0$. By setting $x_{n}=0$ for $n>j+1, x_{0}=0$, the system $\left(c_{j} I-A^{*}\right) x=0$ reduces to a homogeneous linear system of $j$ equations in $j+1$ unknowns, so that nontrivial solutions exist. Therefore $c_{j} I-A \in \mathrm{III}$.

If $c_{J}=\gamma /(2-\gamma)$ then clearly $c_{j} I-A \in 3$. Assume $0<c_{j}<$ $\gamma /(2-\gamma)$ and let $r$ denote the largest integer such that $c_{r}=c_{k}$. Solving $\left(c_{r} I-A\right) x=0$ leads to (7) with $j=r$.

Pick $\varepsilon=\min \left\{\gamma(1-\gamma) /(2-\gamma) 2, \gamma / 2-1 /\left(1+1 / c_{j}\right)\right\}$. (Since $c_{j}<$ $\gamma /(2-\gamma), \gamma / 2-1 /\left(1+1 / c_{j}\right)>0$.) Choose $N$ large enough so that, for $m \geq N, c_{m+j+1}>\gamma-\varepsilon$. From $c_{J}<\gamma /(2-\gamma)$ it follows that

$$
c_{j+m+1} / c_{j}-1>((2-\gamma) / \gamma) c_{j+m+1}-1>((2-\gamma) / \gamma)(\gamma-\varepsilon)-1>0
$$

since $\varepsilon<\gamma(1-\gamma) /(2-\gamma)$.

For $m \geq N$, from (7),

$$
\frac{\left|x_{j+m+1}\right|}{\left|x_{j+m}\right|}=\left|\frac{1-c_{j+m+1}}{1-\frac{c_{j+m+1}}{c_{j}}}\right|=\frac{1-c_{j+m+1}}{\frac{c_{j+m+1}}{c_{J}}-1}<\frac{1-\gamma+\varepsilon}{\frac{\gamma-\varepsilon}{c_{j}}-1}<1,
$$

since $\varepsilon<\gamma-2 /\left(1+1 / c_{j}\right)$. Consequently $\left\{x_{n}\right\} \in l$, hence $\left\{x_{n}\right\} \in c$, and $c_{j} I-A$ is not $1-1$.

Suppose $A$ has a zero on the main diagonal and $\gamma>0$. Let $j$ denote the smallest positive integer for which $c_{j}=0$. Let $e^{j}$ denote the coordinate sequence with a 1 in the $j$ th positive and all other entries zero. Then $A e^{j}=0$, and $c_{j} I-A=-A$ is not $1-1$. By setting $x_{0}=0, x_{n}=0$ for $n>j+1$, the system $\left(c_{j} I-A^{*}\right) x=0$ reduces to a homogeneous linear system of $j$ equations in $j+1$ unknowns.

When the diagonal entries of $A$ do not converge, it was shown in [2] that the spectrum need no longer be a disc. This fact was illustrated by considering weighted mean methods with diagonal entries $c_{0}=1, c_{2 n}=$ $1 / p, c_{2 n-1}=1 / q, n>0$, where $1<p<q$. Under these conditions, $\sigma(A)$ $=\left\{\left.\lambda|(p-1)(q-1)| \lambda\right|^{2} \geq|1-p \lambda||1-q \lambda|\right\}$. The boundary of the spectrum is either an oval, two ovals tangent at a point on the $x$-axis between 0 and 1 , or two disjoint ovals, depending on the relative sizes of $p$ and $q$. It will now be shown that the fine spectra of these methods behave exactly as the fine spectra for the weighted mean methods considered in Theorems 1-5.

THEOREM 6. Let $A$ be a regular weighted mean method defined by $c_{0}=1, \quad c_{2 n}=1 / p, c_{2 n-1}=1 / q, \quad n>0$, where $1<p<q$. If $\lambda \neq$ $1 / p, 1 / q, 1$ and satisfies $(p-1)(q-1)|\lambda|^{2}>|1-p \lambda||1-q \lambda|$, then $\lambda \in \operatorname{III}_{1} \sigma(A)$. 
Proof. Since $\lambda \neq 1 / p, 1 / q$, or $1, \lambda I-A$ is a triangle, so it is $1-1$, and $\lambda I-A \in 1 \cup 2$.

Suppose $\left(\lambda I-A^{*}\right) x=0$. Then, as in the proof of Theorem 1, $x_{0}=0$, $x_{1}$ is arbitrary and, from (2),

$$
\begin{aligned}
x_{2 n} & =\frac{p_{2 n-1}}{p_{0}} x_{1}\left(1-\frac{1}{\lambda}\right)\left(1-\frac{1}{p \lambda}\right)^{n-1}\left(1-\frac{1}{q \lambda}\right)^{n-1}, \\
x_{2 n+1} & =\frac{p_{2 n}}{p_{0}} x_{1}\left(1-\frac{1}{\lambda}\right)\left(1-\frac{1}{p \lambda}\right)^{n-1}\left(1-\frac{1}{q \lambda}\right)^{n} .
\end{aligned}
$$

From the hypotheses on $A$ it follows that

$$
p_{2 n}=\frac{p_{0} p^{n-1} q^{n}}{(p-1)^{n}(q-1)^{n}}, \quad p_{2 n-1}=\frac{p_{0}(p q)^{n-1}}{(p-1)^{n-1}(q-1)^{n}}
$$

so that

$$
\begin{aligned}
\frac{\left|x_{2 n+2}\right|}{\left|x_{2 n}\right|} & =\frac{\left|x_{2 n+1}\right|}{\left|x_{2 n-1}\right|}=\frac{p q}{(p-1)(q-1)}\left|1-\frac{1}{p \lambda}\right|\left|1-\frac{1}{q \lambda}\right| \\
& =\frac{p q}{(p-1)(q-1)} \frac{|1-p \lambda||1-q \lambda|}{p q|\lambda|^{2}} \\
& <\frac{p q}{(p-1)(q-1)} \frac{|\lambda|^{2}(p-1)(q-1)}{p q|\lambda|^{2}}=1 .
\end{aligned}
$$

Consequently there exist nonzero sequences $\left\{x_{n}\right\} \in l$ such that $\left(\lambda I-A^{*}\right) x=0$ and $\lambda I-A^{*}$ is not $1-1$. Therefore $\lambda I-A \in$ III.

It will now be shown that $\lambda I-A^{*}$ is onto. Suppose $y=\left(\lambda I-A^{*}\right) x$, $x, y \in l$. Then $(\lambda-1) x_{0}=y_{0}$ and (4) holds. Solving (4) for $x$ in terms of $y$ yields the matrix $B$, from $x=B y$, with entries as described in the paragraph following (6). $\Sigma_{n}\left|b_{n 0}\right|=1 /|\lambda-1|<\infty$.

$$
\sum_{n=1}^{\infty}\left|b_{n 1}\right|=\frac{p_{1}}{p_{0}|\lambda|}+\sum_{n=3}^{\infty} \frac{p_{n-1}}{p_{0}|\lambda|} \prod_{j=1}^{n-2}\left|1-\frac{c_{j}}{\lambda}\right|
$$


The series on the right can be written in the form $\Sigma_{1}+\Sigma_{2}$, where

$$
\begin{aligned}
\Sigma_{1} & =\sum_{n=1}^{\infty} \frac{p_{2 n}}{p_{0}|\lambda|} \prod_{j=1}^{2 n-1}\left|1-\frac{c_{j}}{\lambda}\right| \\
\Sigma_{2} & =\sum_{n=2}^{\infty} \frac{p_{2 n-1}}{p_{0}|\lambda|} \prod_{j=1}^{2 n-2}\left|1-\frac{c_{j}}{\lambda}\right| . \\
\Sigma_{1} & =\frac{1}{p_{0}|\lambda|} \sum_{n=1}^{\infty} \frac{p_{0} p^{n-1} q^{n}}{(p-1)^{n}(q-1)^{n}}\left|1-\frac{1}{p \lambda}\right|^{n-1}\left|1-\frac{1}{q \lambda}\right|^{n} \\
& =\frac{|q \lambda-1|}{(p-1)(q-1)|\lambda|^{2}} \sum_{n=1}^{\infty} R^{n-1},
\end{aligned}
$$

where $R=|p \lambda-1||q \lambda-1| /(p-1)(q-1)|\lambda|^{2}$.

Similarly,

$$
\Sigma_{2}=\frac{1}{(q-1)|\lambda|} \sum_{n=2}^{\infty} R^{n-1}
$$

From the hypothesis on $\lambda$, both series are convergent geometric series. For $k>1$,

$$
\sum_{n=k}^{\infty}\left|b_{n k}\right|=\frac{1}{|\lambda|}\left(1+\frac{p_{k}}{|\lambda| P_{k-1}}\right)+\sum_{n=k+2}^{\infty} \frac{p_{n-1}}{|\lambda| P_{k-1}} \prod_{j=k}^{n-2}\left|1-\frac{c_{j}}{\lambda}\right| .
$$

Since the above series is dominated by the series in (12), $\|B\|_{1}<\infty$.

Theorem 7. Let $A$ be as in Theorem 6. If $\lambda=1 / p, 1 / q$ then $\lambda \in$ III $_{1} \sigma(A)$.

Proof. Suppose $\lambda=1 / p$. Then $\lambda I-A$ maps $x$ into $\left\{(1 / p-1) x_{0}\right.$, $-a_{10} x_{0}+(1 / p-1 / q) x_{1},-a_{20} x_{0}-a_{21} x_{1},-a_{30} x_{0}-a_{31} x_{1}-a_{32} x_{2}+$ $\left.(1 / p-1 / q) x_{3}, \cdots\right\}$, so that $(\lambda I-A) x=0$ implies $x_{0}=x_{1}=0$. By induction, one solves successively a pair of equations of the form

$$
\begin{aligned}
& -a_{2 n+1,2 n} x_{2 n}+(1 / p-1 / q) x_{2 n+1}=0, \\
& -a_{2 n+2,2 n} x_{2 n}-a_{2 n+2,2 n+1} x_{2 n+1}=0,
\end{aligned}
$$

whose only solution is $x_{2 n}=x_{2 n+1}=0$, since the determinant of the coefficients is equal to $p_{2 n} / p P_{2 n+2} \neq 0$.

If $\lambda=1 / q$, then $\lambda I-A$ maps $x$ into $\left\{(1 / q-1) x_{0},-a_{10} x_{0},-a_{20} x_{0}-\right.$ $\left.a_{21} x_{1}+(1 / q-1 / p) x_{2}, \cdots\right\}$, and $(\lambda I-A) x=0$ forces $x_{0}=0$. Again 
one solves successively pairs of equations, this time of the form

$$
\begin{aligned}
& -a_{2 n+2,2 n+1} x_{2 n+1}+(1 / q-1 / p) x_{2 n+2}=0, \\
& -a_{2 n+3,2 n+1} x_{2 n+1}-a_{2 n+3,2 n+2} x_{2 n+2}=0 .
\end{aligned}
$$

Since the determinant of the coefficients is equal to $p_{2 n+1} / q P_{2 n+3} \neq 0$, $x=0$, and $\lambda I-A$ is $1-1$. Clearly $\lambda I-A \in I I I$, and it remains to show that $\lambda I-A^{*}$ is onto.

Suppose $\left(\lambda I-A^{*}\right) x=y, x, y \in l$. As in the proof of Theorem 2, choosing $x_{j+1}=0$ we can solve for $x_{0}, \cdots, x_{j}$ in terms of $y_{0}, \cdots, y_{j+1}$, and the remaining values of $x$ are determined from $x=B y$, where $B$ is as defined in (8). Since $c_{j+2}=c_{j}$ for each $j>0$, it is clear from (8) that $b_{j+m, j+m-k}=b_{j+m, j+1}=0$ for $k \geq 3, m \geq 4$. Also, $b_{j+m, j+m-2}=0$ for $m$ even. Consequently $B$ has at most three nonzero diagonals, with bounded elements, and $B \in B(l)$.

THEOREM 8. If $A$ is defined as in Theorem 6, and $\lambda$ satisfies

$$
(p-1)(q-1)|\lambda|^{2}=|1-p \lambda||1-q \lambda|, \quad \lambda \neq 1,
$$

then $\lambda \in \mathrm{II}_{2} \sigma(A)$.

Proof. Since $\lambda I-A$ is a triangle, it is $1-1$, so that $\lambda I-A \in 1 \cup 2$. Now consider $\left(\lambda I-A^{*}\right) x=0$. Then, as in the proof of Theorem 1 , $x_{0}=0$ and $x_{n}$ satisfies (2) for $n>0$. It then follows that

$$
\left|x_{2 n}\right|=\frac{\left|x_{1}\right|}{q-1}\left|1-\frac{1}{\lambda}\right|
$$

and

$$
\left|x_{2 n+1}\right|=\frac{\left|x_{1}\right||\lambda-1|}{|p \lambda-1|} .
$$

Therefore $\left\{x_{n}\right\} \in l$ implies $x=0$ and $\lambda I-A^{*} \in 1 \cup 2$. It then follows that $\lambda \in \mathrm{II}_{2} \sigma(A)$.

From Theorem 4 it follows that $1 \in \mathrm{III}_{3} \sigma(A)$.

Cartlidge [1] demonstrated that certain weighted mean methods belong to $B\left(l^{p}\right), p \geq 1$, and computed their spectra. For example, he showed that, if $\delta=\lim p_{n} / P_{n}>0$, then $A \in B\left(l^{p}\right)$ and

$$
\sigma(A)=\left\{\lambda \| \lambda-(2-\delta)^{-1} \mid \leq(1-\delta) /(2-\delta)\right\} \cup S .
$$

It can be shown that the results of Theorems 1-5 are true for each such $A$.

Based on the results established in this paper, the following is a reasonable conjecture. 
Let $A$ be a weighted mean method, $A \in B\left(l^{p}\right)$, for some $p$ satisfying $1 \leq p \leq \infty$. Then all interior points of $\sigma(A)$ belong to III $_{1}$, all boundary points, except 1 , and possibly $\gamma /(2-\gamma)$, belong to $\mathrm{II}_{2}$, and 1 and all isolated points belong to III $_{3}$. If $\gamma /(2-\gamma)$ is a diagonal element of $A$, then $\gamma /(2-\gamma) \in \mathrm{III}_{3}$. Otherwise $\gamma /(2-\gamma) \in \mathrm{II}_{2}$.

\section{REFERENCES}

1. J. P. Cartlidge, Weighted Mean Matrices as Operators on $l^{p}, \mathrm{Ph} . \mathrm{D}$. Dissertation, Indiana University, 1978.

2. Frank P. Cass and B. E. Rhoades, Mercerian theorems via spectral theory, Pacific J. Math., 73 (1977), 63-71.

3. S. Goldberg, Unbounded Linear Operators, McGraw Hill, New York, 1966.

4. K. Knopp, Theory and Application of Infinite Series, Hafner Publishing Co., New York, 1971.

5. Robert B. Wenger, The fine spectra of the Hölder summability operators, Indian J. Pure and Appl. Math., 6 (1975), 695-712.

Received May 1, 1979 and in revised form January 29, 1982.

INDIANA UNIVERSITY

BLOOMINGTON, IN 47405 


\section{PACIFIC JOURNAL OF MATHEMATICS \\ EDITORS}

DONALD BABBITT (Managing Editor)

University of California

Los Angeles, CA 90024

\section{Hugo Rossi}

University of Utah

Salt Lake City, UT 84112

C. C. Moore and Arthur Ogus

University of California

Berkeley, CA 94720
J. DugundiI

Department of Mathematics

University of Southern California

Los Angeles, CA 90089-1113

R. FINN and H. SAMELSON

Stanford University

Stanford, CA 94305

ASSOCIATE EDITORS
R. ARens
E. F. BECKENBACH
B. H. NeumanN
F. WOLF
K. YoshidA (1906-1982)

\section{SUPPORTING INSTITUTIONS}

UNIVERSITY OF ARIZONA

UNIVERSITY OF BRITISH COLUMBIA

CALIFORNIA INSTITUTE OF TECHNOLOGY

UNIVERSITY OF CALIFORNIA

MONTANA STATE UNIVERSITY

UNIVERSITY OF NEVADA, RENO

NEW MEXICO STATE UNIVERSITY

OREGON STATE UNIVERSITY
UNIVERSITY OF OREGON

UNIVERSITY OF SOUTHERN CALIFORNIA

STANFORD UNIVERSITY

UNIVERSITY OF HAWAII

UNIVERSITY OF TOKYO

UNIVERSITY OF UTAH

WASHINGTON STATE UNIVERSITY

UNIVERSITY OF WASHINGTON 


\section{Pacific Journal of Mathematics}

Vol. 104, No. 1

May, 1983

Nestor Edgardo Aguilera and Eleonor Ofelia Harboure de Aguilera, On

the search for weighted norm inequalities for the Fourier transform $\ldots \ldots .1$

Jin Akiyama, Frank Harary and Phillip Arthur Ostrand, A graph and its complement with specified properties. VI. Chromatic and achromatic numbers ......................................... 15

Bing Ren Li, The perturbation theory for linear operators of discrete type . . . 29

Peter Botta, Stephen J. Pierce and William E. Watkins, Linear

transformations that preserve the nilpotent matrices .............. 39

Frederick Ronald Cohen, Ralph Cohen, Nicholas J. Kuhn and Joseph

Alvin Neisendorfer, Bundles over configuration spaces .......... 47

Luther Bush Fuller, Trees and proto-metrizable spaces . . . . . . . . . . 55

Giovanni P. Galdi and Salvatore Rionero, On the best conditions on the

gradient of pressure for uniqueness of viscous flows in the whole space . . 77

John R. Graef, Limit circle type results for sublinear equations $\ldots \ldots \ldots \ldots 85$

Andrzej Granas, Ronald Bernard Guenther and John Walter Lee,

Topological transversality. II. Applications to the Neumann problem for

$y^{\prime \prime}=f\left(t, y, y^{\prime}\right) \ldots \ldots \ldots \ldots \ldots \ldots \ldots \ldots \ldots \ldots \ldots \ldots \ldots \ldots \ldots . \ldots 5$

Richard Howard Hudson and Kenneth S. Williams, Extensions of

theorems of Cunningham-Aigner and Hasse-Evans . . . . . . . . . . . 111

John Francis Kurtzke, Jr., Centralizers of irregular elements in reductive algebraic groups

James F. Lawrence, Lopsided sets and orthant-intersection by convex

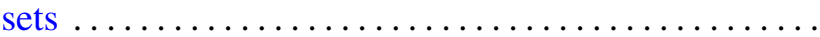

Åsvald Lima, G. H. Olsen and U. Uttersrud, Intersections of $M$-ideals and

$G$-spaces

Wallace Smith Martindale, III and C. Robert Miers, On the iterates of derivations of prime rings

Thomas H. Pate, Jr, A characterization of a Neuberger type iteration procedure that leads to solutions of classical boundary value problems

Carl L. Prather and Ken Shaw, Zeros of successive iterates of multiplier-sequence operators

Billy E. Rhoades, The fine spectra for weighted mean operators

Rudolf J. Taschner, A general version of van der Corput's difference theorem

Johannes A. Van Casteren, Operators similar to unitary or selfadjoint ones 